PROCEEDINGS OF THE

AMERICAN MATHEMATICAL SOCIETY

Volume 137, Number 4, April 2009, Pages 1179-1184

S 0002-9939(08)09646-9

Article electronically published on September 25, 2008

\title{
CONGRUENCE PROPERTIES OF HERMITIAN MODULAR FORMS
}

\author{
TOSHIYUKI KIKUTA AND SHOYU NAGAOKA \\ (Communicated by Wen-Ching Winnie Li) \\ In celebration of Tomoyoshi Ibukiyama's 60th birthday
}

\begin{abstract}
We study the existence of a modular form satisfying a certain congruence relation. The existence of such modular forms plays an important role in the determination of the structure of a ring of modular forms modulo $p$. We give a criterion for the existence of such a modular form in the case of Hermitian modular forms.
\end{abstract}

\section{INTRODUCTION}

In [7, H. P. F. Swinnerton-Dyer determined the structure of a ring of modular forms $\bmod p$ in the elliptic modular case. In his argument, the existence of a certain modular form plays an important role. Namely, he used the fact that there exists a modular form $f$ of weight $p-1$ with $p$-integral Fourier coefficients such that

$$
f \equiv 1 \quad(\bmod p) .
$$

(Also cf. Serre 6. .) In the elliptic modular case, such a form can be constructed easily. In fact, we may take $f=E_{p-1}$ (the normalized Eisenstein series of weight $p-1)$. However, the problem of existence in the case of Siegel modular forms turns out to be difficult. For example, the Siegel-Eisenstein series $E_{p-1}^{(n)}$ of weight $p-1$ is no longer a solution in general. In [2], S. Boecherer and the second author studied this problem and gave some criteria for the existence problem in the case of Siegel modular forms.

In this paper, we give a criterion of the existence problem in the case of Hermitian modular forms over the imaginary quadratic fields $\mathbb{Q}(\sqrt{-1})$ and $\mathbb{Q}(\sqrt{-3})$.

\section{HeRmitian MODUlar FORMS}

We start by recalling the definition of Hermitian modular forms. For details, please refer to $\left[3\right.$. The Hermitian half-space $\mathbb{H}_{n}$ of degree $n$ is defined by

$$
\mathbb{H}_{n}:=\left\{Z \in M_{n}(\mathbb{C}) \mid \frac{1}{2 \sqrt{-1}}\left(Z-{ }^{t} \bar{Z}\right)>0\right\} .
$$

Received by the editors April 1, 2008.

2000 Mathematics Subject Classification. Primary 11F33; Secondary 11F55.

Key words and phrases. Congruences for modular and $p$-adic modular forms.

The second author was supported in part by Grant-in-Aid for Scientific Research 19540061.

(C)2008 American Mathematical Society Reverts to public domain 28 years from publication 
Let $\mathbb{K}$ be an imaginary quadratic field with discriminant $d_{\mathbb{K}}$. We denote by $\mathcal{O}=\mathcal{O}_{\mathbb{K}}$ the ring of integers and by $\mathcal{O}^{\times}$the group of units in $\mathcal{O}$.

The Hermitian modular group of degree $n$ over $\mathbb{K}$,

$$
U_{n}(\mathcal{O}):=\left\{M \in M_{2 n}(\mathcal{O}) \mid{ }^{t} \bar{M} J_{n} M=J_{n}\right\}, \quad J_{n}=\left(\begin{array}{cc}
0 & 1_{n} \\
-1_{n} & 0
\end{array}\right),
$$

acts on $\mathbb{H}_{n}$ by

$$
Z \longmapsto M<Z>:=(A Z+B)(C Z+D)^{-1}
$$

for all $Z \in \mathbb{H}_{n}$ and $M=\left(\begin{array}{ll}A & B \\ C & D\end{array}\right) \in U_{n}(\mathcal{O})$.

Let $\Gamma \subset U_{n}(\mathcal{O})$ be a subgroup of $U_{n}(\mathcal{O})$. A holomorphic function $F(Z)$ on $\mathbb{H}_{n}$ is called a Hermitian modular form of weight $k$ for $\Gamma$ if it satisfies the functional equations:

$$
F(M<Z>)=\operatorname{det}(C Z+D)^{k} F(Z)
$$

for all $Z \in \mathbb{H}_{n}$ and $M=\left(\begin{array}{ll}A & B \\ C & D\end{array}\right) \in \Gamma$. (We assume the holomorphy at the cusps in the case $n=1$.) We denote by $M_{k}(\Gamma)$ the space of Hermitian modular forms of weight $k$ for $\Gamma$. Later we mainly deal with the case $\Gamma=U_{n}(\mathcal{O})$ or $S U_{n}(\mathcal{O}):=$ $U_{n}(\mathcal{O}) \cap S L_{2 n}(\mathcal{O})$. In both cases, $F \in M_{k}(\Gamma)$ has a Fourier expansion of the form

$$
F(Z)=\sum_{0 \leq T \in \Lambda_{n}} a_{F}(T) \exp \{2 \pi \sqrt{-1} \operatorname{tr}(T Z)\},
$$

where $T$ runs over the lattice

$$
\Lambda_{n}=\Lambda_{n}(\mathbb{K}):=\left\{T=\left(t_{i j}\right) \in \operatorname{Her}_{n}(\mathbb{K}) \mid t_{i i} \in \mathbb{Z}, \sqrt{d_{\mathbb{K}}} t_{i j} \in \mathcal{O}\right\}
$$

(cf. [3]).

\section{MAin RESUlt}

In this section, we state the main result of this paper, which gives a criterion on the existence of a modular form satisfying a certain congruence relation.

Let $p$ be a rational prime and $\mathbb{Z}_{(p)}=\left\{\frac{a}{b} \in \mathbb{Q} \mid p \nmid b\right\}$ denote the localization of $\mathbb{Z}$ at $p$. We denote by $M_{k}(\Gamma)_{\mathbb{Z}_{(p)}}$ the subset of $M_{k}(\Gamma)$ consisting of $F \in M_{k}(\Gamma)$ such that all of its Fourier coefficients belong to $\mathbb{Z}_{(p)}$.

Our main result can be stated as follows:

Theorem 3.1. (1) Assume that $\mathbb{K}=\mathbb{Q}(\sqrt{-1})$ or $\mathbb{K}=\mathbb{Q}(\sqrt{-3})$. There exists a Hermitian modular form $F_{p-1} \in M_{p-1}\left(S U_{n}(\mathcal{O})\right)_{\mathbb{Z}_{(p)}}$ such that

$$
F_{p-1} \equiv 1 \quad(\bmod p)
$$

if

$$
p \equiv 1 \quad(\bmod 4) .
$$

(2) Assume that $\mathbb{K}=\mathbb{Q}(\sqrt{-1})$. There exists a Hermitian modular form $F_{p-1} \in$ $M_{p-1}\left(U_{n}(\mathcal{O})\right)_{\mathbb{Z}_{(p)}}$ such that

$$
F_{p-1} \equiv 1 \quad(\bmod p)
$$

if and only if

$$
p \equiv 1 \quad(\bmod 4) .
$$




\section{4. $p$-Special Hermitian matrix}

In order to prove our theorem, we need to consider the existence of a $p$-special Hermitian matrix.

A Hermitian matrix $H={ }^{t} \bar{H} \in \operatorname{Her}_{m}(\mathbb{K})$ is called $p$-special if $H$ satisfies the following four conditions:

(i) $H$ is positive definite,

(ii) $H$ is even integral, namely, $H \in 2 \Lambda_{m}$,

(iii) $\operatorname{det} H=\left(\frac{2}{\sqrt{\left|d_{\mathbb{K}}\right|}}\right)^{m}$,

(iv) (the main condition) there exists a $p$-group $C_{p}$ in the finite unitary group

$$
U_{m}(H ; \mathcal{O}):=\left\{U \in M_{m}(\mathcal{O}) \mid{ }^{t} \bar{U} H U=H\right\}
$$

such that the group $C_{p}$ acts freely on $\mathcal{O}^{m} \backslash\{\mathbf{0}\}$.

Our proof of the main result mainly depends on the existence of a $p$-integral Hermitian matrix. To demonstrate the existence of such a matrix we use the result of Bayer-Fluckiger, which guarantees the existence of a suitable even unimodular lattice over $\mathbb{Z}$.

Theorem 4.1 (Bayer-Fluckiger [1]). Let $m$ be a positive integer such that $m$ is not a power of 2 . Then there exists a definite unimodular lattice having an automorphism with characteristic polynomial $\Phi_{m}$ if and only if $m$ is mixed and $\varphi(m)$ is divisible by 8. Here $\Phi_{m}$ is the $m$-th cyclotomic polynomial.

Our result in this section is as follows:

Proposition 4.2. Assume that $\mathbb{K}=\mathbb{Q}(\sqrt{-1})$ or $\mathbb{Q}(\sqrt{-3})$. If $p$ is a prime number such that $p \equiv 1(\bmod 4)$, then there exists a p-special Hermitian matrix $H$ of rank $p-1$.

Proof. First we assume that $\mathbb{K}=\mathbb{Q}(\sqrt{-1})$ and $p \equiv 1(\bmod 4)$. If we put $m=4 p$, then $m$ is not a prime power and $\varphi(m)=2(p-1)$ is divisible by 8 . Hence, by Theorem 4.1, there exists an even unimodular positive definite lattice $(L, S)$ having an automorphism with characteristic polynomial $\Phi_{m}$ ( $m$-th cyclotomic polynomial), where $S$ is the associated bilinear form. We denote by $t$ such an automorphism. The order of the automorphism $t^{p}$ is 4 , and $L$ becomes a $\mathbb{Z}[\sqrt{-1}]$-module by identifying $\sqrt{-1}$ with $t^{p}$. Since $\mathbb{Z}[\sqrt{-1}]$ is principal, one may construct a $\mathbb{Z}[\sqrt{-1}]$-basis of $L$. Hence $L$ becomes a $\mathbb{Z}[\sqrt{-1}]$-lattice of rank $p-1$. (The $\mathbb{Z}$-rank of $L$ is $\varphi(m)=$ $2(p-1)$.) The corresponding Gram matrix $H$ is the desired matrix. Indeed, one can confirm that the Hermitian matrix $H$ satisfies the conditions (i)-(iv) of the $p$-special Hermitian matrix.

(i) The positivity of $H$ comes from that of $L$.

(ii) The bilinear form $S: L \times L \longrightarrow \mathbb{Z}$ satisfies

$$
S(\boldsymbol{x}, \boldsymbol{y})=\frac{1}{2}(h(\boldsymbol{x}, \boldsymbol{y})+h(\boldsymbol{y}, \boldsymbol{x})),
$$

where $h: L \times L \longrightarrow \mathbb{Q}(\sqrt{-1})$ is the Hermitian form associated with $H$. Since $h(\boldsymbol{x}, \boldsymbol{x})=S(\boldsymbol{x}, \boldsymbol{x}) \in 2 \mathbb{Z}, H$ is even integral. 
(iii) If we denote by $B \in \operatorname{Sym}_{2(p-1)}(\mathbb{Z})$ the Gram matrix associated with the bilinear form $S$, then we have

$$
\operatorname{det} B=(\operatorname{det} H)^{2} \cdot\left(\frac{\sqrt{\left|d_{\mathbb{K}}\right|}}{2}\right)^{2(p-1)} .
$$

Since $\operatorname{det} B=1$ and $\left|d_{\mathbb{K}}\right|=4$, we have $\operatorname{det} H=1$. This shows that the matrix $H$ satisfies the condition (iii).

(iv) We recall the definition of the automorphism $t$ mentioned above. In this case, the $p$-group $C_{p}:=<t^{4}>$ acts freely on $\mathcal{O}^{p-1} \backslash\{\mathbf{0}\}$ because the characteristic polynomial of $t$ is $\Phi_{m}$.

Next we assume that $\mathbb{K}=\mathbb{Q}(\sqrt{-3})$ and $p \equiv 1(\bmod 4)$. If we put $m=3 p$, then, by a similar argument to that stated above, there exists an even unimodular positive definite lattice $(L, S)$ having an automorphism with characteristic polynomial $\Phi_{m}$. We denote by $s$ such an automorphism. The order of the automorphism $s^{p}$ is 3 , and $L$ becomes an $\mathcal{O}_{\mathbb{K}}$-module by identifying $\omega=\frac{-1+\sqrt{-3}}{2}$ with $s^{p}$. Since $\mathcal{O}_{\mathbb{K}}=$ $\mathbb{Z}+\omega \mathbb{Z}$ is principal, one may construct an $\mathcal{O}_{\mathbb{K}}$-basis of $L$. One can prove that the corresponding Gram matrix satisfies the conditions of the $p$-special Hermitian matrix in a way similar to the case $\mathbb{K}=\mathbb{Q}(\sqrt{-1})$. This completes the proof of Proposition 4.2.

Example 4.3. We give examples of $H$ in the case $p=5$ :

The case $\quad \mathbb{K}=\mathbb{Q}(\sqrt{-1}), \quad H=\left(\begin{array}{cccc}2 & 0 & 1+\sqrt{-1} & \sqrt{-1} \\ 0 & 2 & \sqrt{-1} & 1-\sqrt{-1} \\ 1-\sqrt{-1} & -\sqrt{-1} & 2 & 0 \\ -\sqrt{-1} & 1+\sqrt{-1} & 0 & 2\end{array}\right)$.

The case $\quad \mathbb{K}=\mathbb{Q}(\sqrt{-3}), \quad H=\left(\begin{array}{cccc}2 & 0 & \frac{2}{\sqrt{-3}} & \frac{2}{\sqrt{-3}} \\ 0 & 2 & \frac{2}{\sqrt{-3}} & \frac{-2}{\sqrt{-3}} \\ \frac{-2}{\sqrt{-3}} & \frac{-2}{\sqrt{-3}} & 2 & 0 \\ \frac{-2}{\sqrt{-3}} & \frac{2}{\sqrt{-3}} & 0 & 2\end{array}\right)$.

\section{Proof of the Main theorem}

In this section, we prove our main theorem.

Proof. (1) We assume that $\mathbb{K}=\mathbb{Q}(\sqrt{-1})$ or $\mathbb{K}=\mathbb{Q}(\sqrt{-3})$ and that $p \equiv 1$ $(\bmod 4)$. By Proposition 4.2, there exists a $p$-special Hermitian matrix $H$ of rank $p-1$. We denote by $C_{p}$ the corresponding $p$-group (cf. section 4 , the definition of a $p$-special Hermitian matrix (iv)). We associate the theta series

$$
\vartheta_{H}(Z):=\sum_{X \in M_{p-1, n}(\mathcal{O})} \exp \{\pi \sqrt{-1} \operatorname{tr}(H[X] Z)\}, \quad Z \in \mathbb{H}_{n},
$$

where $H[X]:={ }^{t} \bar{X} H X$. The modularity of $\vartheta_{H}$ for $S U_{n}(\mathcal{O})$ comes from the conditions (i), (ii), and (iii) of the $p$-special matrix $H \in 2 \Lambda_{p-1}$ (e.g. cf. Cohen and Resnikoff [4], p. 332); namely, we have $\vartheta_{H}(Z) \in M_{p-1}\left(S U_{n}(\mathcal{O})\right)$. In particular, we have $\vartheta_{H}(Z) \in M_{p-1}\left(U_{n}(\mathcal{O})\right)$ in the case that $\mathbb{K}=\mathbb{Q}(\sqrt{-1})$ because 
$\sharp \mathcal{O}^{\times}=\sharp \mathbb{Z}[\sqrt{-1}]^{\times}=4$ and the weight $p-1$ is divisible by 4 . The Fourier expansion is given as follows:

$$
\begin{aligned}
& \vartheta_{H}(Z)=\sum_{T} A(H, T) \exp \{2 \pi \sqrt{-1} \operatorname{tr}(T Z)\}, \\
& A(H, T)=\sharp \mathcal{A}(H, T), \quad \mathcal{A}(H, T)=\left\{X \in M_{p-1, n}(\mathcal{O}) \mid H[X]=2 T\right\} .
\end{aligned}
$$

If $T \neq O_{n}$, then the $p$-group $C_{p}$ acts freely on the set $\mathcal{A}(H, T)$. Therefore, the number $A(H, T)$ is divisible by $p$. Since $A\left(H, O_{n}\right)=1$, we have

$$
\vartheta_{H}(Z) \equiv 1 \quad(\bmod p) .
$$

This proves (1) of Theorem 3.1.

(2) Assume that $\mathbb{K}=\mathbb{Q}(\sqrt{-1})$ and that there exists a form

$$
F_{p-1} \in M_{p-1}\left(U_{n}(\mathbb{Z}(\sqrt{-1}))\right)_{\mathbb{Z}_{(p)}}
$$

such that

$$
F_{p-1} \equiv 1 \quad(\bmod p)
$$

We recall the definition of the $\Phi$-operator defined by

$$
\Phi: M_{k}\left(U_{n}(\mathcal{O})\right) \longrightarrow M_{k}\left(U_{n-1}(\mathcal{O})\right), \quad \Phi(F)(Z):=\lim _{\lambda \rightarrow \infty} F\left(\left(\begin{array}{cc}
Z & 0 \\
0 & i \lambda
\end{array}\right)\right), Z \in \mathbb{H}_{n-1}
$$

If we apply the $\Phi$-operator $n-1$ times to $F_{p-1}$, then

$$
\Phi^{(n-1)}\left(F_{p-1}\right) \in M_{p-1}\left(U_{1}(\mathbb{Z}[\sqrt{-1}])\right)_{\mathbb{Z}_{(p)}}
$$

still satisfies the congruence relation

$$
\Phi^{(n-1)}\left(F_{p-1}\right) \equiv 1 \quad(\bmod p) .
$$

If $p \not \equiv 1(\bmod 4)$, this is impossible because

$$
M_{k}\left(U_{1}(\mathbb{Z}[\sqrt{-1}])\right)=\left\{\begin{array}{l}
M_{k}\left(S L_{2}(\mathbb{Z})\right) \text { if } \quad k \equiv 0 \quad(\bmod 4), \\
0 \quad \text { otherwise. }
\end{array}\right.
$$

(This comes from the fact that $U_{1}(\mathcal{O})=\mathcal{O}^{\times} \cdot S L_{2}(\mathbb{Z})$.) We have proved the statement (2), thereby completing the proof of Theorem 3.1.

\section{REMARK}

In the case that $\mathbb{K}=\mathbb{Q}(\sqrt{-1})$ and $n=2$, there is another construction of $F_{p-1}$, which is based on the theory of Hermitian Jacobi forms.

We assume that $\mathbb{K}=\mathbb{Q}(\sqrt{-1})$. Freitag [5] constructed a set of generators of the graded ring

$$
M^{\text {sym }}\left(U_{2}(\mathbb{Z}[\sqrt{-1}])\right)=\bigoplus M_{k}^{\text {sym }}\left(U_{2}(\mathbb{Z}[\sqrt{-1}])\right),
$$

where $M_{k}^{s y m}\left(U_{2}(\mathbb{Z}[\sqrt{-1}])\right)$ is the subspace consisting of the symmetric Hermitian modular forms of weight $k$. (In general, $F \in M_{k}\left(U_{n}(\mathcal{O})\right.$ ) is called symmetric if $F\left({ }^{t} Z\right)=F(Z)$.) We recall the weight 4 generator $\varphi_{4}$ of $M^{\text {sym }}\left(U_{2}(\mathbb{Z}[\sqrt{-1}])\right.$ ) (cf. [5]). It is known that all the Fourier coefficients of $\mathcal{E}_{4}:=\frac{1}{4} \varphi_{4}$ are integral and the constant term is equal to 1 . We expand $\mathcal{E}_{4}$ as a Fourier-Jacobi series and take the index 1 Jacobi form $\Phi_{4,1}$. All of the Fourier coefficients of $\Phi_{4,1}$ are divisible by 240 . We put $\phi_{4,1}:=\frac{1}{240} \Phi_{4,1}$. Now we assume that $p \equiv 1(\bmod 4)$. Then

$$
f_{p-1,1}:=E_{4}^{\frac{p-5}{4}} \cdot \phi_{4,1}
$$


becomes a Hermitian Jacobi form of weight $p-1$ and index 1 . Here $E_{4}=1+$ $240 \sum_{n=1}^{\infty} \sigma_{3}(n) q^{n}$ is the ordinary Eisenstein series of weight 4 for $S L_{2}(\mathbb{Z})$. All of the Fourier coefficients of $f_{p-1,1}$ are integral and the constant term is equal to 1 . We consider the Maass lift $\mathcal{M}_{k}$ from the space of Hermitian Jacobi forms of weight $k$ and index 1 to the space $M_{k}\left(U_{2}(\mathbb{Z}[\sqrt{-1}])\right)$. Then

$$
F_{p-1}:=-\frac{2(p-1)}{B_{p-1}} \mathcal{M}_{p-1}\left(f_{p-1,1}\right)
$$

is the desired form, namely, $F_{p-1} \in M_{p-1}\left(U_{2}(\mathbb{Z}[\sqrt{-1}])\right)_{\mathbb{Z}_{(p)}}$ and

$$
F_{p-1} \equiv 1 \quad(\bmod p) \text {. }
$$

(Since the Maass lift $\mathcal{M}_{k}$ is defined only for $k$ such that $k \equiv 0(\bmod 4)$ in this case, we need the assumption $p \equiv 1(\bmod 4)$.)

\section{ACKNOWLEDGEMENT}

The authors wish to thank Prof. G. Nebe for suggesting the proof of Proposition 4.2 .

\section{REFERENCES}

1. E. Bayer-Fluckiger, Definite unimodular lattices having an automorphism of given characteristic polynomial. Comment. Math. Helv. 59 (1984), 509-538. MR.780074 (86k:11032)

2. S. Boecherer, S. Nagaoka, On mod p properties of Siegel modular forms. Math. Ann. 338 (2007), 421-433. MR2302069 (2008d:11041)

3. H. Braun, Hermitian modular functions. Ann. of Math. (2) 50 (1949), 827-855. MR0032699 $(11: 333 \mathrm{a})$

4. D. M. Cohen, H. L. Resnikoff, Hermitian quadratic forms and Hermitian modular forms. Pac. J. Math. 76 (1978), 329-337. MR506135 (80b:10039)

5. E. Freitag, Modulformen zweiten Grades zum rationalen und Gausschen Zahlkörper. Sitzungsber. Heidelberger Akad. Wiss. Math.-Natur. Kl. (1967), 3-49. MR0214541 (35:5391)

6. J-P. Serre, Formes modulaires et fonctions zêta p-adiques. Modular Functions of One Variable III, Lecture Notes in Math. 350 (1973), 191-268, Springer. MR0404145 (53:7949a)

7. H. P. F. Swinnerton-Dyer, On $\ell$-adic representations and congruences for coefficients of modular forms. Modular Functions of One Variable III, Lecture Notes in Math. 350 (1973), 1-55, Springer. MR0406931 (53:10717a)

Department of Mathematics, Kinki University, Higashi-Osaka, Osaka 577-8502, Japan

E-mail address: kikuta@math.kindai.ac.jp

Department of Mathematics, Kinki University, Higashi-Osaka, Osaka 577-8502, Japan

E-mail address: nagaoka@math.kindai.ac.jp 